\title{
Ganglion Cyst of the Sternoclavicular Joint in an Adult
}

\author{
Nam Gyun Kim, \\ Yun Sub Lim, \\ Jae Hoon Choi, \\ Jun Sik Kim, \\ Kyung Suk Lee \\ Department of Plastic and Reconstructive \\ Surgery, Institute of Health Sciences, \\ Gyeongsang National University Hospital, \\ Gyeongsang National University School of \\ Medicine, Jinju, Korea
}

No potential conflict of interest relevant to this article was reported.

\begin{abstract}
Ganglion cysts are most common on the dorsum of the hand or wrist, but they can occur in any part of the body. There have been few papers reporting ganglion cysts originating from the sternoclavicular joint, with most of these cases developing in children. A 76-year-old woman was referred to our department because of a painless mass over the right sternoclavicular joint. The mass was excised along with the portion of the sternoclavicular joint capsule surrounding the stalk. Histopathologic examination showed the cyst wall to be composed of compressed collagen fibers without evidence of an epithelial or synovial lining, which was consistent with ganglion cyst. To our knowledge, this is the first report of such a cyst in an adult. We consider this to be a useful report for surgeons that treat mass lesions occurring in almost any part of the body surface.
\end{abstract}

Keywords: Ganglion cysts / Joint capsule / Sternoclavicular joint

\section{INTRODUCTION}

Ganglion cysts usually arise close to joints and tendons. The cysts are attached to an underlying joint capsule or tendon sheath by a narrow stalk. Most often they are encountered over the dorsum of the hand or wrist, but they can occur in any part of the body, wherever periarticular or peritendinous connective tissue is present.

We report a ganglion cyst originating from the sternoclavicular joint of an adult. To our knowledge, there have been only two papers that reported ganglion cysts of the sternoclavicular joint $[1,2]$. However, most of these cases involved children, and one of the two papers did not focus on the ganglion cyst but on serous synovitis of the sternoclavicular joint. Therefore, this is the first report of a ganglion cyst of the sternoclavicular joint developing in an adult.

\section{Correspondence: Kyung Suk Lee}

Department of Plastic and Reconstructive Surgery, Institute of Health Science, Gyeongsang National University College of Medicine, Gyeongsang National

University Hospital, 79 Gangnam-ro, Jinju 660-702, Korea

E-mail: opensound@hanmail.net

Received November 27, 2013 / Revised February 18, 2014 / Accepted February 26, 2014

\section{CASE REPORT}

A 76-year-old woman was referred to our department because of a painless mass over the right sternoclavicular joint. The lesion was first recognized 3 months earlier. It was felt to have slightly increased in size. There was no history of trauma or evidence of diabetes, hypertension, or rheumatoid arthritis. On physical examination, the mass was found to be firm, round, smooth, and 1.5×1.5 $\mathrm{cm}$ in size in the subcutaneous tissue. The mass was not tender to palpation, and it was firmly fixed to the sternoclavicular joint. The overlying skin and subcutaneous tissue was freely movable. Neither ultrasonography, computed tomography, nor magnetic resonance imaging of the sternoclavicular joint was performed.

Excisional biopsy revealed that, intraoperatively, the wall of the cyst was thin, firmly elastic, and sharply outlined, and it contained amorphous, gelatinous material (Fig. 1). The stalk was directed toward the sternoclavicular joint. The mass was excised along with the portion of the sternoclavicular joint capsule surrounding the stalk. Histopathologic examination showed the cyst wall to be composed of compressed collagen fibers without evidence of an 
epithelial or synovial lining, which was consistent with ganglion cyst (Fig. 2). There has been no evidence of recurrence 11 months postoperatively.

\section{DISCUSSION}

Ganglion cysts are most common on the upper extremities such as the dorsal or volar aspect of the wrist, flexor tendon sheath, and distal interphalangeal joint of fingers. Less commonly, they have been reported to arise about the foot, the popliteal fossa, and the shoulder. However, there have been few papers reporting ganglion cysts of the sternoclavicular joint, with most of these cases devel-

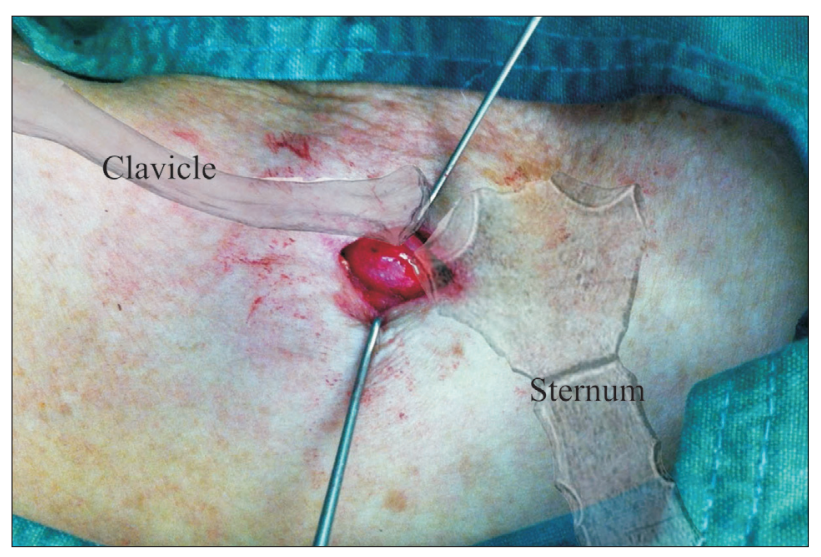

Fig. 1. Intraoperative view showing that the cyst over the right sternoclavicular joint had a thin, firmly elastic, and sharply outlined wall, containing amorphous, gelatinous material.

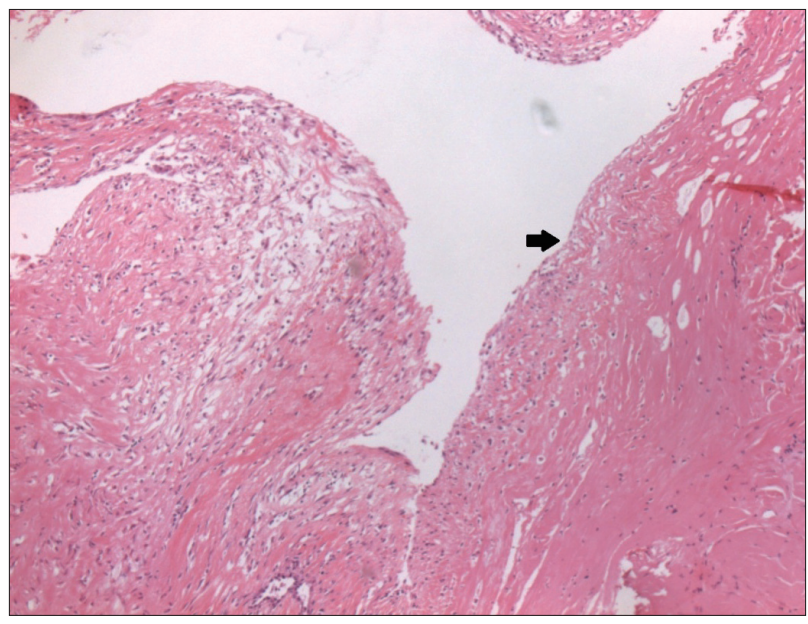

Fig. 2. Photomicrograph showing that the wall of the mass consisted of fibrous tissue (black arrow) without a definite cellular lining $(\mathrm{H} \& \mathrm{E}, \times 40)$. oping in children [1,2]. Haber et al. [1] reported five children with a ganglion cyst of the sternoclavicular joint (aged from 13 months to 9 years). About 30 years ago, Kofoed et al. [2] reported on the differential diagnostic aspects of 17 patients with spontaneous swelling of the sternoclavicular joint, of which two were found to have a ganglion cyst. However, the results were limited because they devoted most of the space to an explanation of serous synovitis, and the two patients with a ganglion cyst were not considered in the details of their demographics [2]. Of their 17 patients, the 10 with serous synovitis were adults, and the rest had ganglion cysts (2), osteomyelitis (2), osteoarthrosis (1), metastasis (1), and Friedrich's disease (1). Of these, three were children. Therefore, there was a high probability that the two patients with ganglion cysts were children.

Our differential diagnosis for the cystic mass of the sternoclavicular joint in adults includes synovial cyst. Synovial cysts and ganglion cysts are morphologically similar because both are filled with viscoid fluid; in the past, these terms have been erroneously considered to be synonymous. However, their histologic features are different. Ganglion cysts have a fibrous tissue lining, whereas synovial cysts have a lining with cuboidal or flattened cells consistent with a synovial origin. Further, synovial cysts around involved joints are common in patients with rheumatoid arthritis. To our knowledge, there have been two papers that reported synovial cysts originating from the sternoclavicular joint in adults, in which all patients had rheumatoid arthritis $[3,4]$.

Surgical excision is the most effective method of treatment for ganglion cysts. Excision of the main cyst only, without the connecting joint capsule, has resulted in a recurrence rate of $30 \%$ to $40 \%$ [5]. Since the adoption of complete excision with removal of a small portion of the joint capsule at the base of the stalk, recurrence rates have improved significantly. Our excision, which included the portion of the sternoclavicular joint capsule surrounding the stalk, obtained a favorable result.

\section{REFERENCES}

1. Haber LH, Waanders NA, Thompson GH, Petersilge C, Ballock RT. Sternoclavicular joint ganglion cysts in young children. J Pediatr Or- 
thop 2002;22:544-7.

2. Kofoed H, Thomsen P, Lindenberg S. Serous synovitis of the sternoclavicular joint. Differential diagnostic aspects. Scand J Rheumatol 1985;14:61-4.

3. Grelier JM, Hardin JG. Letter: Synovial cyst of sternoclavicular joint as mediastinal mass. Ann Intern Med 1975;83:525-6.

4. Angelides AC, Wallace PF. The dorsal ganglion of the wrist: its patho- genesis, gross and microscopic anatomy, and surgical treatment. J Hand Surg Am 1976;1:228-35.

5. Andonopoulos AP, Meimaris N, Yiannopoulos G, Pastromas V, Dimopoulos P. Large synovial cysts originating from the sternoclavicular joints in a patient with rheumatoid arthritis. Ann Rheum Dis 2003;62:1119-20. 